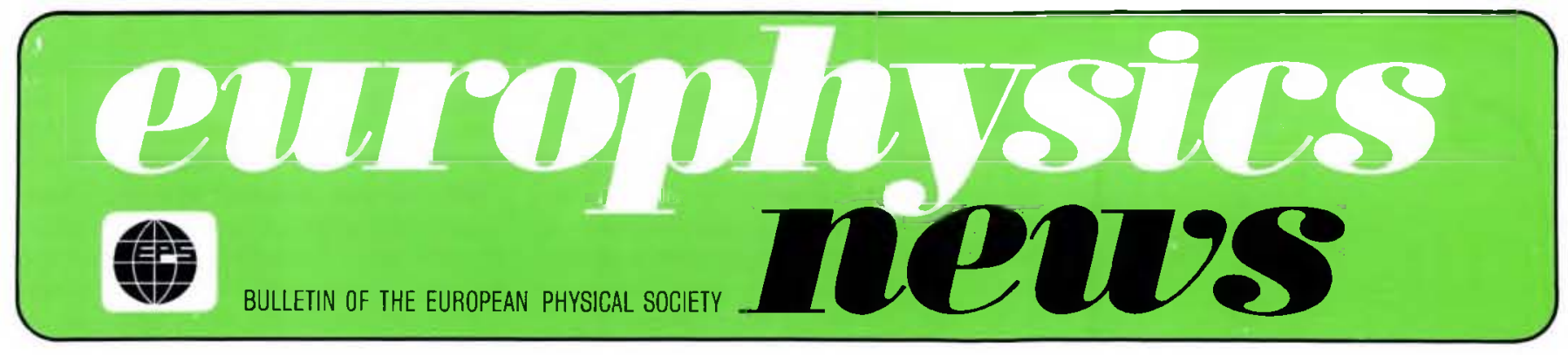

\title{
Gamma-Ray Astronomy Beyond the COS-B Mission
}

\author{
G.F. Bignami, Milan \\ (CNR Laboratorio di Fisica Cosmica e Tecnologie Relative)
}

Photons represent our principal source of information on the Universe, and by collecting them over a few millennia mankind has accumulated the present body of astronomical knowledge. Up to a few decades ago, this was based on the narrow optical band, i.e. the spectral interval matched both by response of the human eye and the transparency of the Earth's atmosphere. The investigation of other, higher energy regions of the e. m. spectrum, shielded by the atmosphere (luckily for all terrestrial life forms) had to await the developments of the space age, and the availability of orbiting detectors. Gamma-ray astronomy is directed towards the most energetic of these extraterrestrial photons, and is the youngest branch of the new astronomies. Here we shall consider it in the photon energy interval between a few tens of $\mathrm{MeV}$ and a few $\mathrm{GeV}$, where most of the data are now available. The photon wavelengths at these energies are small compared to the inter-atomic distances in solids, so that standard focussing optics cannot be employed and each photon arrival direction has to be treated individually, a situation more akin to high energy particle physics than to traditional astronomy. Moreover, even if the $\gamma$-ray photon energy density in the Universe (and in our Galaxy) is a nonneglible $10^{4} \mathrm{eV} / \mathrm{cm}^{3}$, or a fraction $10^{-3}, 10^{4}$ of the optical and infrared and $10^{-1}-10^{-2}$ of the $X$-ray intervals, the photon number flux itself is small $\left(\sim 10^{4}-10^{-5} \mathrm{ph} / \mathrm{s} \mathrm{cm}^{2}\right.$ sr), requiring large collector areas and long exposure times.

In spite of these intrinsic difficulties, as well as of others of an experimental nature, the motivations for doing gamma-ray astronomy are so strong that the observational effort was spurred on through the sixties and seventies, with finally now, rewarding results. These motivations are connected with the obviously non-thermal nature for processes emitting $\geq 100 \mathrm{MeV}$ photons, both in galactic and extragalactic objects (e. g. pulsars and Active Galactic Nuclei) and with the study of the general magnetohydrodynamic equilibrium of the disc of our Galaxy with its gas, magnetic field, and cosmic ray content. It is also important to notice that $\gamma$-ray photons travel unscattered and unabsorbed through great lengths of galactic and intergalactic space, whereas most other windows of astronomy suffer from one form or another of absorption. Because of sensitivity limits, $\gamma$-ray astronomy is so far confined mostly to within our Galaxy, and in what follows mostly galactic data will be reviewed. However, limited but important extragalactic results are available, and they will also be included.

Traditionally, $\gamma$-ray astronomy is also thought to include the lower energy region, with photon energies around $1 \mathrm{MeV}$, brid ging the gap towards $X$-rays, as well as the astronomy of a class of transient events, called " $\gamma$-ray bursts". These will not be treated here, as the large body of results now being accumulated in these two fields has started to represent a fascinating topic per se. In the present region of interest, most of the available information has been gathered by a few space missions, and of them the last and most significant has been the European Space Agency's COS-B. It was put into orbit in August 1975 and was terminated on 25 April 1982, after an un precedented longevity and scientific data output.

The present work is in fact dedicated to the memory of the COS-B satellite, and to

\section{CARAVANE COLLABORATION}

1. Cosmic Ray Working Group, Huygens Laboratorium, Leiden, The Netherlands

2. Istituto di Fisica Cosmica del CNR, in Milan, Italy

3. Istituto di Fisica Cosmica e Informatica del CNR, Palermo, Italy

4. Max-Planck-Institut für Extraterrestrische Physik, Garching bei München, F.R.G.

5. Service d'Electronique Physique, Centre d'Etudes Nucléaires de Saclay, France

6. Space Science Department of the European Space Agency, ESTEC, Noordwijk, The Netherlands

the nearly seven years of $\gamma$-ray data it made available to the European 'Caravane' collaboration (see panel) which has been responsible for the project since its early days, in the late sixties.

Observational Approach: Telescopes for High-Energy Astronomy

Beyond the intrinsic problem of placing a large collector area in orbit for a long time, further experimental difficulties have to be surmounted when designing a $\gamma$-ray telescope. First, the number of $\gamma$-ray photons coming in from the Universe is not only small in absolute terms, but also about five orders of magnitude smaller than the number of high-energy charged particles, mostly protons, in the cosmic ray beam. $\gamma$-rays must therefore be identified within high background conditions. Second, in order to perform an astronomical observation, once each photon has been reliably

\section{Contents}

Gamma-ray Astronomy Beyond the CoS-B Mission

Permanent Magnets

Mixed Valence Compounds of the Rare Earths

EPS Associate Members

Statement of European Physicists 12 


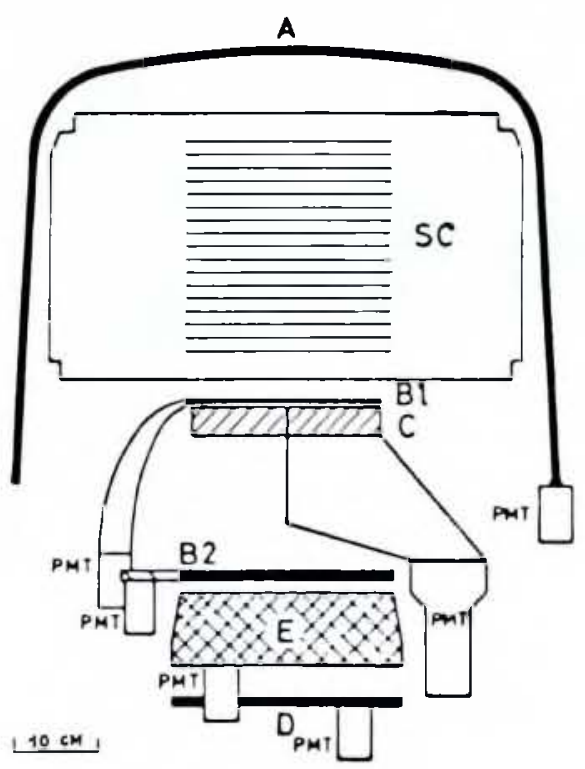

Fig. 1 - Cross-section of the COS-B detector showing the relative positions and scale of the spark chamber (SC), triggering scintillators ( $B^{\prime}$ $\left.B_{2}\right)$ and Cherenkov counter $(C)$, the anticoincidence shield $(A)$ and the energy calorimeter $(E$, $D$ ).

identified, its arrival direction should be known precisely.

As already mentioned, common focussing optics is not applicable to photon energies $\geq 100 \mathrm{MeV}$, and they must be detected individually through their interaction with matter, which in this energy range is in the pair production regime. The detection tool most commonly used is the spark chamber (or, more correctly, a stack of spark chambers), originally borrowed from accelerator high energy physics. The advantage is that the absorbing material can be divided into relatively thin plates so that the paths of the positron and electron generated by the $\gamma$-ray can be followed through the gaps. This permits a sure identification of the event, through its characteristic pattern, and a rough determination of the arrival direction from the bisector of the angle between the $\mathrm{e}^{+}-\mathrm{e}^{-}$tracks. However, not only is this angle rendered uncertain by the inevitable scattering of the charged electrons in the absorbing material, but also by the amount of momentum carried away by the nucleus in the field of which the pair production happens, and which in all practical situations is unmeasurable. In the end, the uncertainties involved, sum up to something of the order of one degree (depending on the photon energy) which is a rather heavy handicap for doing astronomy. Naturally, good statistics, i.e. high photon numbers, can improve these limitations due to the basic physics of the process; on the other hand, other experimental errors (e. g. spacecraft attitude reconstitution) also come into play

A schematic example of a spark chamber $\gamma$-ray detector is given in Fig. 1 which represents an outline of the COS-B European Space Agency experiment. A plastic anticoincidence dome surrounds a sixteengap spark chamber, the firing of which is triggered by a counter coincidence arrangement defining the field of view of the instrument. A high-Z crystal of several radiation lengths thickness is placed under the triggering telescope and acts as a "total" absorption calorimeter to measure the particle (and photon) energy. Before flight, the instrument must be calibrated in a tagged $\gamma$-ray beam for measuring its sensitive area, energy resolution and, above all, angular resolution, or "Point Spread Function". Fig. 2 gives an idea of these quantities for the COS-B experiment. The subsequent mission involves a stabilized spacecraft, orientable in the sky, so that a given target or region of the sky can be observed for a given time and sufficient statistics collected.

The space missions flown so far, devoted to $\gamma$-ray astronomy, include two early NASA satellites, OSO 11 and SAS 2 (in 1968 and 1973 respectively), and ESA's COS-B. Before these missions, a few pioneering balloon flights and a small experiment aboard the Explorer $X \mid$ satellite had proved the existence of an extra-terrestrial flux of hard $\gamma$-radiation.

The "100000 photon" COS-B mission was put on a highly eccentric orbit, as this permitted long, uninterrupted observation periods, few restraints on the coverage of the celestial sphere, little or no effect from the atmospheric albedo and real-time data recovery at a very high level ( $\geq 90 \%$ ) from the ESA ground stations, thus avoiding the need for an on-board tape recorder. The disavantages of such an orbit are the limited telemetry rate, the continuous passage through the Van Allen belts, and the exposure to the bulk of the cosmic ray protons in the energy range $1-10 \mathrm{GeV}$. Satellites operating in a low circular equatorial orbit (as e. g. the SAS-2 satellite) are protected from these background-inducing particles which are deflected away by the Earth's magnetic field, and also remain below the Van Allen belts for most of the orbit. On the other hand, their observational flexibility is limited by the presence of the nearby Earth's atmosphere, and the need for equatorial data recovery is a practical problem for European observers.

\section{The Gamma-Ray Sky}

The first results of gamma-ray astronomy (in the late sixties) gave a picture of the high-energy gamma-ray sky quite different from that at other wavelengths (e. $\mathrm{g}$. optical or X-ray). The dominating feature is the disc of our Galaxy, with a fairly continuous, apparently diffuse emission and with a significant brightening towards the galactic centre. Another type of diffuse emission, significantly weaker yet firmly detected, was observed to originate from the whole sky with no measurable anisotropies.
The first feature could easily be explained in terms of interactions between the cosmic rays (CR) and the Inter-Stellar Matter (ISM) through the $p-p$ interactions, vielding among others $\pi^{\circ}$ mesons, subsequently decaying into two $\gamma$-rays of about $70 \mathrm{MeV}$ each. It was recognized that certain quantitative problems could exist with this model, especially when trying to account for the increased $\gamma$-ray flux from the central galactic regions, due to our lack of independent knowledge of the galactic CR distribution. As for the isotropic emission, it was quickly understood that at least a part of it could be of an extragalactic nature, and a variety of interpretative models were presented which were based both on large scale diffuse cosmological processes and on the sum of individual faint sources, indistinguishable by the instruments. It was mostly the SAS-2 mission, with its very low intrinsic background and its capability of measuring $\gamma$-photons down to $\sim 35 \mathrm{MeV}$, that gave results on this topic, and the current interpretative debate, very much alive, still uses that excellent data base.

As for the disc of our Galaxy, the statistics collected by COS- $B$ have given rise to a significant advance, settling a few points but raising new problems. The picture of the galactic disc is now available with the maximum sharpness possible for current $\gamma$-ray astronomy instruments; it presents a richness of detail. Fig. 3 shows such a picture, divided in three energy ranges because of the energy-dependent instru-

Fig. 2 - Effective sensitive area, angular resolution end energy resolution of the COS.B gamma-ray detector for gamma rays incident parallel to the axis of the experiment and which satisfy the selection requirements applied in the analysis.

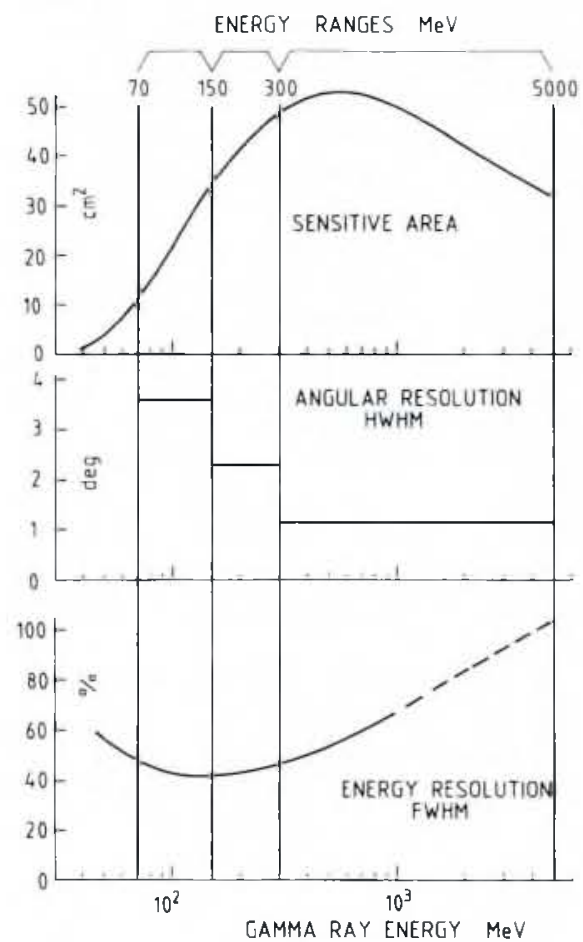




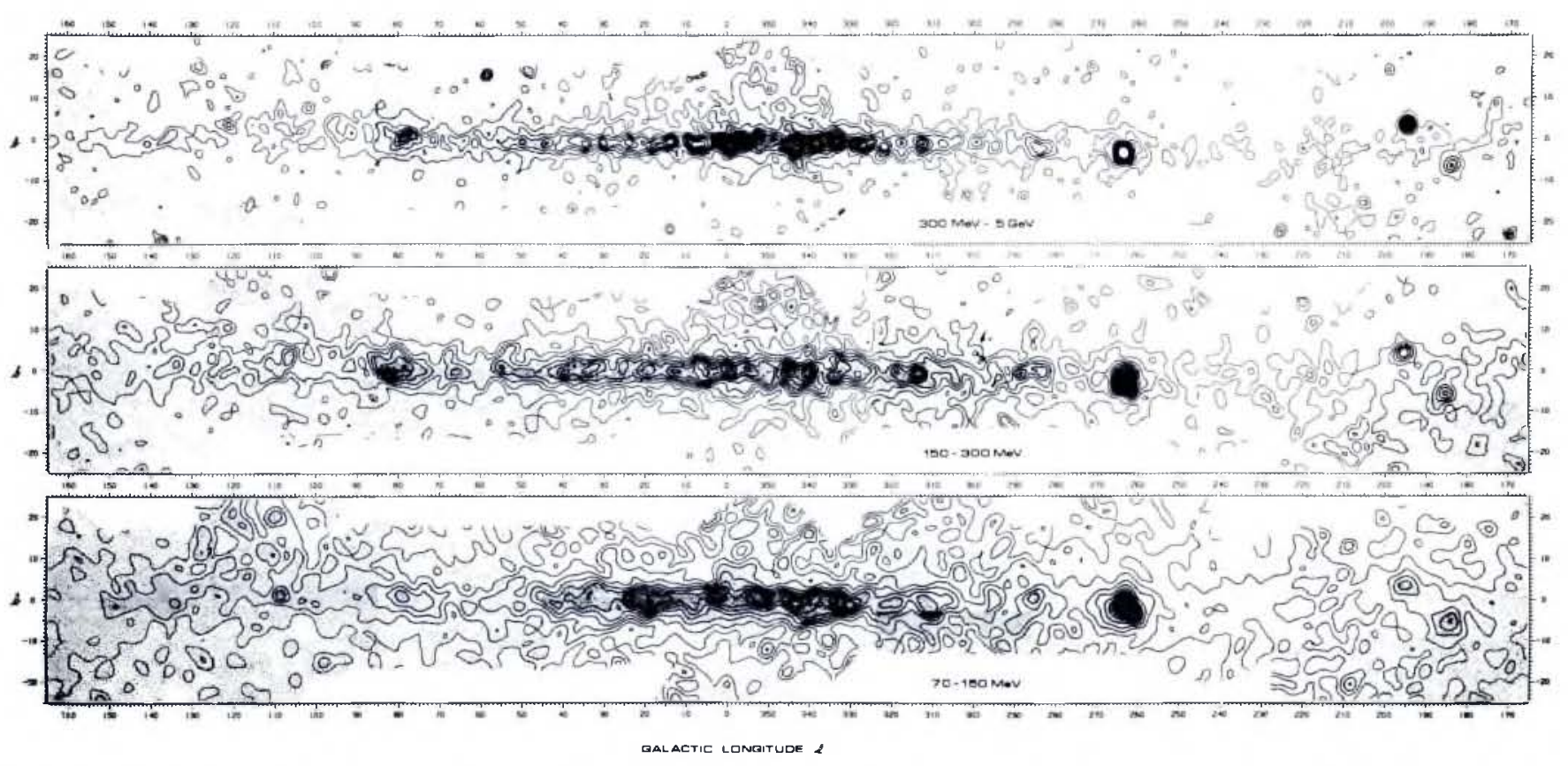

Fig. 3 - Presentation in galactic coordinates of the structure of the galactic gamma-ray emission as measured by COS-B.

mental Point Spread Function. The energy spectrum of the radiation is also available: by and large, it appears not to vary significantly with galactic longitude and also to be well approximated by a power law, i. e. without the expected "bump" at $~-70$ $\mathrm{MeV}$, which would have represented the signature of the $\pi^{0}$ decay process. This can only imply that, if CR-ISM interactions are important, then electrons play a significant role via the bremsstrahlung or inverse Compton processes (the latter involving the various galactic photon fields), both fully capable of generating continuous, powerlaw type spectra.

As for the cartography of the $\gamma$-ray Galaxy in Fig. 3, it shows a great deal of structure of varying angular extent, and this can very well be correlated with our present knowledge of the Milky Way disc as it is traced by other, independent measurements of the ISM.

First, one notes a broad correlation with the very large scale structure or "grand design" of our Galaxy, i. e. with the spiral structure present on a scale of several kpc length: the asymmetry of the profile between latitude $\sim 0^{\circ}-50^{\circ}$ and $\sim 360^{\circ}-$ $310^{\circ}$, the "galactic hole" at $\sim 60^{\circ}$, the peaks in Cygnus $\left(-80^{\circ}\right)$ and Carina ($284^{\circ}$ ), are all features present in, e. g., radio astronomical studies of the spiral structure. Second, a correlation on the "medium" distance scale $(1-3 \mathrm{kpc})$ is apparent in the Cassiopeia-Cepheus-Perseus region $\left(-100^{\circ}-150^{\circ}\right)$. This also is well in agreement with radio maps, both related to the emission of neutral hydrogen and of interstellar molecules. Finally, a strong correlation exists also with the local $(<1 \mathrm{kpc})$ matter, giving rise to medium-latitude gamma-ray emission: the well-known Gould Belt of stars and ISM is seen from
Orion to Ophiucus, as well as other local structures.

The detailed correspondence between the $\gamma$-ray Galaxy and that known through other ISM tracers can only mean that emission from the ISM is important (if not dominant locally), as it had been originally foreseen, although the emission mechanism is not completely understood yet. However, one of the discoveries of the COS-B emission has been that of the presence, along the galactic ridge, of localized enhancements of emission of smaller angular scale than any other structure: the gamma-ray sources. How much of the galactic $\gamma$-ray luminosity is due to these sources, i. e. to a process basically different from that of the diffuse ISM-CR interaction is presently an open question: a Solomonic 50-50 appears to many to be an acceptable compromise.

\section{Unidentified Gamma Objects (UGO's),} Other Cosmic Gamma-Ray Sources.

A separate chapter in the description of the $\gamma$-ray sky must be devoted to the $\gamma$-ray "sources" that are seen against (or on top of) the diffuse radiation. Traditionally, a "source" or, better, a "point source", in astronomy is an object which cannot be resolved by the power of the telescope used, i.e. the true angular extent of which cannot be measured since it is comparable to or smaller than the angular resolution of the telescope. Thus, to ground-based optical telescopes, with typical "seeing" limitation of the order of one arc s, a star in the Galaxy appears as unresolved, whereas frequently nebulae and external galaxies can be seen to exhibit a measurable extent. Precisely the same concept is used in gamma-ray astronomy when we define a celestial gamma-ray source as a significant excess of photons lagainst the background) with arrival direction distribution compatible with the instrument's Point Spread Function. As expected, all the uncertainties connected with the arrival direction of each $\gamma$-ray are reflected into the sky, and anything smaller than about $\sim 1^{\circ}$, depending somewhat on its energy spectrum, cannot be resolved in $\gamma$-ray light. This makes the job of identifying the entity responsible for the $\gamma$-ray emission very difficult, as a huge number of celestial objects, both galactic and extragalactic, are crammed in few square degrees of sky. However, one powerful tool can provide a solution to this problem: the time variability of the emission. Clearly, if the $\gamma$-rays are modulated in time with a law identical to that present at some other wavelength for a known object, the identification of the $\gamma$-ray source is immediate.

The $\gamma$-ray emission from the two fastest free radio pulsars in the Galaxy was discovered in this way by the SAS-2 mission, after some preliminary balloon results. The two pulsars PSR $0532+21$ and $0833-45$ in the constellations of Taurus and Vela are believed to be neutron stars spinning at 30 and $-10 \mathrm{~Hz}$ and are physically associated with the remnants of the supernova explosions by which they were also generated.

The $\gamma$-ray emission evidence from these two objects has led to new insight into their physics, in view of the fact that the power emitted above $100 \mathrm{MeV}\left(\sim 10^{28}\right.$ and $\sim 10^{27}$ $\mathrm{J} / \mathrm{s}$ respectively) is a good fraction (up to a few per mil) of the total energy loss of the pulsars and thus of the energy funnelled by the neutron stars in electromagnetic radiation. A further challenge to any theoretical interpretation is provided by the fact that the two $\gamma$-ray "light curves" for the Crab 
and Vela pulsars match perfectly, whereas they are widely different at other wavelengths (e. g. optical and radio). A variety of models exist for the $\gamma$-ray emission from neutron stars, which can be classified according to the proposed region of emission in, e. g. polar gap, outer gap, light cylinder and wind zone models (in centrifuge order). A special case to consider is that of the source $\mathrm{Cyg} X-3$, where a young pulsar bound in a binary system could also provide the required energy in gamma-rays to account for the flux reported during the SAS-2 mission.

If $\gamma$-ray emission is important for pulsars, it is also logical to ask whether pulsars may be important for the $\gamma$-ray emission from the Galaxy. The answer is that while individually they represent excellent candidate $\gamma$-ray sources, there does not exist a correlation between the $\gamma$-ray emission from the Galaxy (both in diffuse form and in unresolved sources) and the known population of radio pulsars. For the reasons mentioned above, the Crab and Vela pulsars represent the only examples where absolute certainty can be reached for the identification of a $\gamma$-ray source. However, in the panorama of $\gamma$-ray sources they represent a small fraction: while the SAS-2 recognised a total of four or five sources, the much longer COS-B mission has yielded five times as many, listed in the second CoS-B catalogue (see Fig. 4). While the majority of these sources have to be labelled UGO's (Unidentified Gamma Objects), notable exceptions exist.

The source named 2CG $353+16$ (from its galactic coordinates) includes in its error box a dense cloud of interstellar matter, located near the star named " $\rho$ " of the constellation Ophiucus. Given the relatively small distance to this cloud $(\leq 150 \mathrm{pc}$, or $\left.\sim 5 \times 10^{18} \mathrm{~m}\right)$ and given the mass estimates of its gaseous content $1 \leqslant 6000$ solar masses, or $\sim 10^{34} \mathrm{~kg}$ ) obtainable through other independent measurements, it is easy to estimate its $\gamma$-ray flux, assuming for their production the same CR-ISM interaction mechanism as for the diffuse emission. The result is indeed not far from that observed, but a difference remains which can only be accounted for with a far-reaching assumption: the $\gamma$-ray yield of the cloud near $\rho$ Oph must be enhanced relative to the rest of the local ISM, and the simplest way to do so is certainly to postulate a quasi-local, in situ, CR acceleration mechanism. This special yield appears to be a property just of this cloud, since copious other measurements exist by COS-B of the local ISM that are perfectly explainable with the standard galactic $\gamma$-ray "emissivity" and which trace the matter distribution in structures already known at other wavelengths. In fact, another local cloud complex, in Orion, is seen clearly in the COS-B $\gamma$-ray data: it does not appear in the 2CG catalogue simply because, unlike $\rho O p h$, its emission is resolved (and actually mapped) owing to its wide angular extent.

From the very local to the very distant, the only other example of identified COS-B source is 2 CG $289+64$, which contains in its error box the quasar $3 \mathrm{C} 273$, which has a redshift of 0.154 , and thus is believed to be at a distance of $~-900 \mathrm{Mpc}$ for a reasonable value of the Hubble constant. This represents the only extragalactic object ever seen in this energy range; at such a distance, the observed flux implies a luminosity of the order of a few $\times 10^{39} \mathrm{~J} / \mathrm{s}$ in $\gamma$-rays, or an energy output similar for larger) than that emitted in the optical, IR, UV or X-ray ranges. Considering the variability observed for this object at these wavelengths, and the consequent source dimension (derived from the well-known light-travel argument) of $\sim 10^{14} \mathrm{~m}$, it is obvious that some very infernal machine must be at work inside $3 \mathrm{C} 273$ for packing so much energy in so little a volume. In fact, the only plausible mechanism invoked is connected to accretion onto a massive 1 $10^{8}$ solar masses) black hole, the Schwarzschild radius of which, however, is only

Fig. 4 - Region of the sky searched for gamma-ray sources (unshaded) and sources detected above $100 \mathrm{MeV}$ by spatial analysis. The closed circles denote sources with measured fluxes above $1.3 \times$ $10^{-6}$ photon $/ \mathrm{cm}^{2}$ s. Open circles denote sources below this threshold.

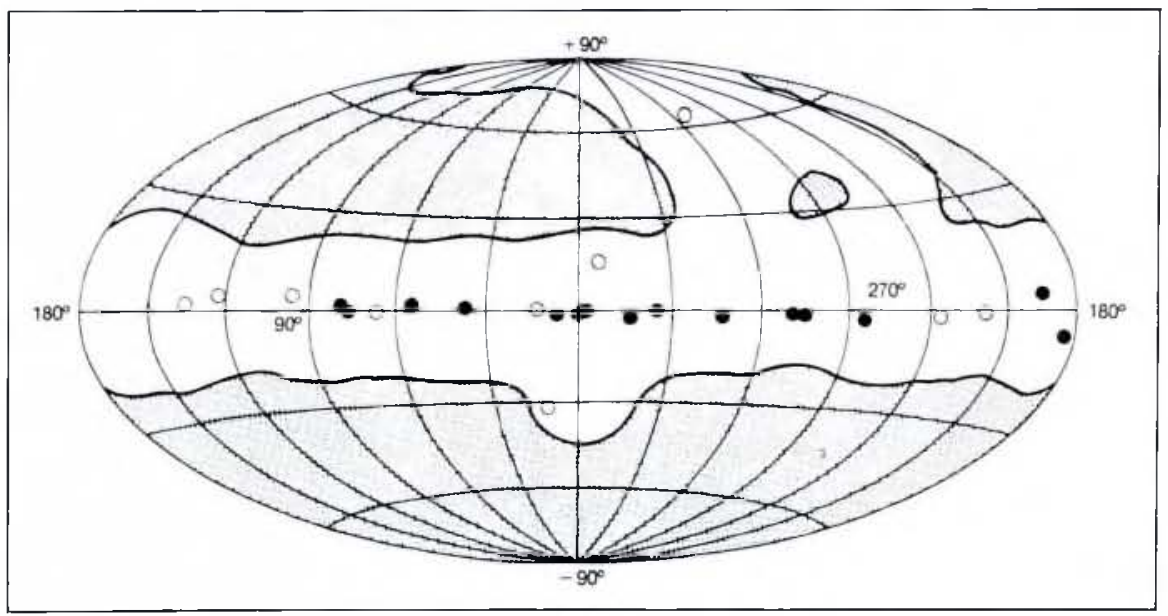

slightly smaller than $10^{14} \mathrm{~m}$. Naturally, it is also very interesting to speculate on a cosmological scale about the consequences of assuming that all quasars emit $\gamma$-rays in a manner similar to the prototype 3C273: the diffuse extragalactic flux first observed by OSO III and confirmed by SAS- 2 could possibly be accounted for as the sum of many faint individual contributions.

Coming back to our Galaxy, the majority of the objects seen in Fig. 4 are UGO's, in the sense that they do not appear to have an obvious counterpart and, moreover, their main electromagnetic emission appears to take place in the $\gamma$-ray range. This is easily seen if one considers that a gamma ray source has an error box in the sky lof surface between 0.6 and 7 square degrees), from which comes an energy flux ranging from 4 to $20 \times 10^{13} \mathrm{~J} / \mathrm{m}^{2} \mathrm{~s}$, in the range from $100 \mathrm{MeV}$ to $1 \mathrm{GeV}$ and for an "average" $E^{\cdot 2}$ differential photon number spectrum. For the sake of comparison, the same amount of energy emission in the 2 - $10 \mathrm{keV}$ interval would imply an X-ray source of roughly between 20 and 100 "Uhuru Flux Units", or a very bright X-ray source indeed. (In the optical $V$ band this corresponds to apparent magnitudes between 7 and 10$)$.

It is urgent to check that no correspondence exists between the COS-B sources and the bright $X$-ray sources in the Galaxy: in the context of high-energy astronomy it is appropriate to refer to them as $\gamma$-ray objects. For the understanding of their nature, two different ways are being tried: one is to study their collective properties and the other is to search in depth for individual counterparts. As for the first, since the COS-B $\gamma$-ray sources do form a new galactic population, their average properties can be investigated, such as galactic latitude, distance and luminosity, as well as their longitude distribution and numberflux $(\log N-\log S)$ relation. The result is that the UGO's are certainly related to a very young galactic population (as, e. g., molecular clouds, OB stars etc), loosely connected with the Galaxy's spiral structure and are probably characterized by a galactocentric gradient rather similar to that of the known supernova remnants.

As for the individual searches, they have been tried in some detail and consistency at two frequencies, in radio and $X$-rays. In the first case, some of the major radiotelescopes in the world have been used to search a few of the UGO error boxes, looking mostly for undiscovered pulsars (especially fast ones) with the hope then to succeed in synchronizing the $\gamma$-ray data with the pulsar timing signature. Work is still in progress on this topic, both within and outside the Caravane collaboration where no one is in the least discouraged by the first negative results. For the $X$-ray search, a logical adjacent window to explore, 
NASA's Einstein Observatory has been extensively used. Through the unprecedented powers of this $X$-ray telescope, several new $X$-ray sources have been discovered in connection with UGO's. However no firm, one-to-one association can be claimed as yet. Here again, work is still in progress, with special emphasis on the 2CG $195+4$ source, discovered by SAS-2 and dubbed "Geminga" by the COS-B workers, some of whom are now pondering on the preliminary intriguing $X$-ray data of the Geminga enigma.

\section{Future Prospects for Gamma-Ray As- tronomy}

With the last of the $\gamma$-ray telescopes just passed away, it is natural to enquire on the future steps to take for advancing highenergy astrophysics. Theoretically, there is a serious need for a better understanding of the UGO's as well as of the emission from pulsars and AGNs; the problem here would seem to be the absence of discrimination between the large variety of models proposed, or alternatively, with the lack of one sufficiently accurate model. Observations at other wavelengths are crucial, but at the same time not easy. The understanding of the diffuse emission from the ISM and of that from local structures and molecular clouds seems to be pinned down reasonably well at the moment, to the point that this aspect of $\gamma$-ray astronomy is now considered a new tool for the tracing of the diffuse galactic matter.

Besides better theoretical work however, clearly better instuments are also needed, and the direction to go is that of improving the angular resolution, definitely the main current $\gamma$-ray astronomy limitation. A lot can be achieved by designing better spark chambers of the "classic" type so as to minimize the basic physics effects responsible for the angular uncertainty and at the same time decrease the background. An instrument of this type, also characterized by a very large sensitive area, is being planned for the NASA Gamma-Ray Observatory by a U.S.-German collaboration, and will fly before the end of the eighties. A different approach is used by the Soviet-FrancoItalian mission 'Gamma 1', to be flown hopefully in less than two years time. It uses a "random" mask collimation technique, aiready applied succesfully in other branches of astronomy as well as in ground-based, non-astronomical equipment. An angular resolution of $-1-5$ arc min on a few bright sources could be obtained, thus tremendously reducing the sizes of the UGO error boxes and the corresponding number of candidate counterparts. Because of its nature, this mission can be considered as complementary to GRO, and eventually from the joint data base it should be possible to make a solid step forward in this energetic window on the Universe.

\title{
PERMANENT MAGNETS
}

\section{Characterization and Materials Development}

\author{
Ch. Gnehm, Lupfig, $\mathrm{CH}$ \\ (Ugimag Recoma $A G$ )
}

The ferromagnetic materials, which are characterized by their very high magnetic permeability $\mu$, may be clearly divided into two groups: the magnetically soft (easy to magnetize and demagnetize) and the magnetically hard (hard to magnetize and demagnetize). Even in the absence of an initially applied external magnetic field, both groups of material posses their own magnetic field. In both, the ease of magnetization and therefore the behaviour under load, is determined by the structure of the magnetic domains whereas the magnitude of the saturation magnetization is not structure-sensitive, but depends on the phase of the material.

In soft magnetic materials, the boundaries of the magnetic domains need to move as freely as possible which is achieved by avoiding trapping by impurities, crystal or grain boundaries, strain centres etc. The job of this group of materials is chiefly the multiplying of magnetic flux in electromagnetic machines and devices. The other group, the magnetically hard materials, find their use as bearers for magnetic recordings or as permanent ma gnets. After the initial magnetization, a permanent magnet must be able to resist the effects of demagnetizing fields, including its own, so that high stability of the magnetic domains is a primary requirement. In other words, domain walls should not move and the polarity of the domains should remain in the original state.

\section{Working Principle of Permanent Ma- gnets}

In the following, we shall confine our attention to the permanent magnets. They are of great importance as industrial products and find their application in a wide variety of electro-mechanical transducer systems. These are typically high performance electric motors, generators, positioning and holding devices, instruments, microwave and focussing tubes, loudspeakers, bearings, clutches; we find permanent magnets in household products, consumer goods, light and heavy industrial machinery, car equipment, precision instruments, telecommunications, data processing, aircraft and space equipment.

In all these practical cases, the magnet itself is fundamentally an energy-storage device which does no net work on its surroundings. Its only function is to provide an external magnetic field. Therefore, it must have free poles. In other words, a magnet always operates on open circuit. The efficiency of a permanent magnet system is very sensitive to the demagnetization curve of the permanent magnet material under consideration, more specifically to the intersection of the load line OP $10=$ origin, $\mathrm{P}=$ operating point) with this curve. Fig. 1 shows the demagnetization curve as the second quadrant part of the hysteresis loop of a permanent magnet in an external ma gnetic field $H$. The slope of the load line OP is equal to $-\left(4 \pi-N_{d}\right) / N_{d}$ where $N_{d}$ (demagnetizing factor) is defined by the length / diameter ratio of the magnet in a fixed position.

The demagnetizing field $H_{\mathrm{d}}=N_{\mathrm{d}} \times M$ ( $M=$ magnetization) is one of the most fundamental parameters in any open permanent magnet circuit systern. This is owing to the fact that the permeability $\mu$ defined as the extent to which an originally unmagnetized body disturbs a magnetic field into which it is placed - is always the result of an interaction between the outside field or the field in the air gap and the field created inside the magnet.

The designer of a permanent magnet system usually attempts to make the best use of the available materials by choosing operating points with maximum product $B \times H$ i.e. $(B H)_{\max }$ but as many applications deal not only with static open circuits, but also with dynamic conditions, the internal self-demagnetizing field will not always remain constant and so the slope of the load line will change. In this case, the demagnetization curve should approximate a straight line with maximum coercive field. In practice, however, the choice of material and design is more complicated by other requirements such as mechanical, thermal and environmental stability, optimum weight and volume, and of course by material and manufacturing costs, which in turn may vary considerably as a function of ductility or machinability and other physical properties.

\section{Historical Development of Materials}

The history of permanent magnets starts with the compass needle, which was already in use in early Chinese culture. The first synthetic permanent magnets were obtained by rubbing a piece of iron with the naturally occurring magnetite $\mathrm{Fe}_{3} \mathrm{O}_{4}$, also called lodestone. In the early $19^{\text {th }}$ century, the experiments and theories of Oersted, Ampère, Faraday and their contemporaries resulted in the construction of the first 